\title{
OBTENÇÃO DE PARÂMETROS DE DESFOSFORAÇÃO DE FERRO-GUSA EM DIFERENTES TEMPERATURAS DE PROCESSO*
}

\author{
Cynara Christ Klippe/ ${ }^{1}$ \\ Camila Santos Scopel ${ }^{2}$ \\ Anna Paula Littig Berger ${ }^{3}$ \\ Daniela Bahiense de Oliveira 4 \\ Felipe Costa Broseghini ${ }^{5}$ \\ José Roberto de Oliveira ${ }^{6}$
}

\section{Resumo}

Devido às exigências de teores de fosforo cada vez menores no aço, o processo de desfosforação tem ganhado destaque nas indústrias. Para aproveitamento das melhores condições termodinâmicas a etapa mais eficiente para o processo de desfosforação ocorre no ferro-gusa. Esse trabalho utilizou quatro misturas iniciais de composição definida com base na literatura, juntamente com o ferro-gusa para ser simulado o processo de desfosforação. A simulação foi feita através do software de cálculos termodinâmicos FactSage e através dos resultados obtidos do programa foram feitos cálculos para avalição da eficiência do processo. Além disso, também foram feitos cálculos baseados na literatura para comparação de resultados e estudo mais aprofundado dos resultados. Os resultados obtidos comprovaram a teoria inicial de que menores temperaturas favorecem o processo.

Palavras-chave: Desfosforação; Ferro-gusa; Termodinâmica computacional.

\section{HOT METAL DEPHOSPHORIZATION PARAMETERS GATHERING IN DIFFERENT TEMPERATURES OF THE PROCESS}

\begin{abstract}
Due to lower phosphor content demands in steel, the dephosphorization process has begun to be highlighted among the industries. For the best use of thermodynamic conditions, the most efficient step in the dephosphorization process occurs in the pig iron. This research utilized 4 initial mixtures with composition defined based on the literature, along with the hot metal, so we could simulate the dephosphorization process. The simulation was performed using a thermodynamic calculation software called FactSage and through the yielded results, calculations were made to evaluate the process efficiency. Besides that, calculations based on literature were also made to compare the results and to perform a deeper study of the results. The yielded results proved the initial theory that lower temperatures facilitate the process.
\end{abstract}

Keywords: Dephosphorization; Hot Metal; Computational thermodynamics.

1 Engenheira Metalurgista e Mestranda em Engenharia Metalúrgica e de Materiais, IFES, Vitória, ES, Brasil, cynarachrist@hotmail.com.

2 Graduanda em Engenharia Metalúrgica, IFES, Vitória, ES, Brasil, camilascopel@hotmail.com.

3 Engenheira Metalurgista e Mestranda em Engenharia Metalúrgica e de Materiais, IFES, Vitória, ES, Brasil,annaplittig@gmail.com.

4 Graduanda em Engenharia Metalúrgica, IFES, Vitória, ES, Brasil, danielabahiense@hotmail.com.

5 Engenheiro Metalurgista e Mestre em Engenharia Metalúrgica e de Materiais, Instituto Federal do Espírito Santo, Vitória, ES, Brasil,felipe.broseghini@gmail.com.

6 Prof. Dr. do Instituto Federal do Espírito Santo, IFES, dos cursos de graduação e pós-graduação em Engenharia Metalúrgica e de Materiais, Vitória, ES, Brasil,jroberto@ifes.edu.br. 


\section{INTRODUÇÃO}

A exigência do baixo teor de fósforo no aço atualmente se deve ao fato de que o mesmo interfere em propriedades importantes do aço como sua tenacidade e resistência, principalmente nos aços com altos teores de carbono, prejudicando assim a sua utilização em várias aplicações. Para diminuir o teor de fósforo no aço, utiliza-se o processo de desfosforação (DeP).

A desfosforação pode ser feita em diferentes etapas do processo de fabricação do aço, porém é mais eficiente e proveitoso que a mesma seja realizada no ferro-gusa, devido às condições termodinâmicas apresentadas nessa etapa serem mais favoráveis, além de evitar a projeção de escória para fora do convertedor, o que acontece quando a DeP é feita no mesmo. Por isso a desfosforação do ferro-gusa se mostra uma alternativa interessante para atingir os teores desejáveis de fósforo no aço.

O processo de DeP, independentemente de onde seja realizado, é feito através da oxidação do fósforo do metal, podendo ser representada pela (Equação 1) abaixo:

$$
2[\mathrm{P}]+5[\mathrm{O}]=\left(\mathrm{P}_{2} \mathrm{O}_{5}\right)
$$

Para isso são usados desfosforantes. Os desfosforantes devem possuir uma fonte de oxigênio, utilizada para a oxidação do fósforo e outra fonte de $\mathrm{CaO}$ responsável pela fixação do pentóxido de fósforo e também pela diminuição do coeficiente de atividade do $\mathrm{P}_{2} \mathrm{O}_{5}$, tornando a reação de desfosforação efetiva e retardando a refosforação do ferro gusa. Segue abaixo a reação de oxidação do fósforo com o oxigênio fornecido a partir do óxido de ferro presente na escória (Equação 2) e a reação entre o $\mathrm{CaO}$ com o pentóxido de fósforo (Equação 3).

$$
\begin{aligned}
& 2[\mathrm{P}]+5(\mathrm{FeO})=\left(\mathrm{P}_{2} \mathrm{O}_{5}\right)+5[\mathrm{Fe}] \\
& \left(\mathrm{P}_{2} \mathrm{O}_{5}\right)+3(\mathrm{CaO})=\left(3 \mathrm{CaO} \cdot \mathrm{P}_{2} \mathrm{O}_{5}\right)
\end{aligned}
$$

Segue ainda a equação (Equação 4) proveniente da soma das duas anteriores:

$$
2[\mathrm{P}]+5(\mathrm{FeO})+3(\mathrm{CaO})=\left(3 \mathrm{CaO} . . \mathrm{P}_{2} \mathrm{O}_{5}\right)+5[\mathrm{Fe}]
$$

Este trabalho propõe-se a avaliar a melhor forma operacional de realizar a desfosforação do gusa, avaliando o parâmetro temperatura e sua influencia no processo de forma a otimiza-lo, atribuindo ao aço os teores de fósforo desejáveis. De acordo com Turkdogan [1], os principais fatores que influenciam o processo são descritos na seguinte equação (Equação 5), que é proveniente de cálculos matemáticos realizados a partir da (Equação 4):

$$
\ln (\% P)_{e q}=\frac{1}{2} \ln \left(a_{3 \mathrm{CaO} . P 2 O 5}\right)+\frac{\Delta G^{0}}{2 R T}-\frac{5 \mu_{O 2}}{4 R T}-\frac{3}{2} \ln \left(a_{C a O}\right)-\ln \left(f_{p}\right)
$$

Sendo assim, analisando a equação, podemos perceber que as baixas temperaturas favorecem os menores teores de fósforo.

Ainda temos a reação de velocidade da desfosforação que é influenciada pelo transporte de massa do fosforo no metal e na escoria. Segue a equação: 


$$
-\frac{d[\% P]}{d t}=k^{\prime} \frac{A \rho_{m}}{W_{m}}([\% P]-[\% P e q])
$$

Onde: $\mathrm{k}^{\prime}=$ Coeficiente de transferência de massa global $(\mathrm{m} / \mathrm{s}) ; \rho_{\mathrm{m}}=$ Densidade do metal $\left(\mathrm{kg} / \mathrm{m}^{3}\right) ; A=$ Área interfacial $\left(\mathrm{m}^{2}\right) ; \mathrm{W}_{\mathrm{m}}=$ Massa de metal $(\mathrm{kg}) ;[\% \mathrm{P}]=$ porcentagem de fósforo no metal; [\%Peq]: porcentagem de fósforo de equilíbrio no metal. Esse fator leva em consideração a atividade do $\mathrm{CaO}$ e $\mathrm{FeO}$, a fração da fase líquida e a porcentagem de $\mathrm{CaO}$ sólido nas misturas. É de grande importância o fator pois a quantidade de $\mathrm{CaO}$ sólido nas misturas iniciais interfere na saturação da escória no final do processo, por outro lado essa quantidade não deve ser muito grande, para que no final se tenha a maior quantidade de escória líquida.

\section{MATERIAIS E MÉTODOS}

A realização desse trabalho foi dividida basicamente em duas etapas que foram:

- Estudo das misturas iniciais no software de termodinâmica

- Estudo do equilíbrio do gusa com a mistura inicial em software de termodinâmica

\subsection{Estudo das misturas iniciais}

As composições iniciais das misturas está apresentada na tabela (Tabela 1) abaixo, já o ferro-gusa tem a seguinte composição: $94,66 \% \mathrm{Fe} ; 0,005 \% \mathrm{~S} ; 4,45 \% \mathrm{C} ; 0,32 \% \mathrm{Si}$; $0,12 \% \mathrm{P}$ e $0,39 \% \mathrm{Mn}$. As composições foram obtidas através de estudos anteriores e testes experimentais realizados por Broseghini [2]. Através dessas foram realizados os aquecimentos da mistura inicial e escória final no software FactSage 6.4 usando como banco de dados: FtOxid e FactPS e como sub banco de dados o Slag? e Pure solids, para as temperaturas $\mathrm{T} 1=1350^{\circ} \mathrm{C}, \mathrm{T} 2=1400^{\circ} \mathrm{C}$ e $\mathrm{T} 3=1450^{\circ} \mathrm{C}$.

Tabela 1. Composição das misturas [2]

\begin{tabular}{ccccccccc}
\hline \multirow{2}{*}{ Mistura } & \multicolumn{9}{c}{ Composição (\%) } & & Relação & Massa da \\
& $\mathrm{FeO}$ & $\mathbf{C a O}$ & $\mathrm{SiO}_{2}$ & $\mathrm{Al}_{2} \mathbf{O}_{3}$ & $\mathbf{M g O}$ & MnO & CaO/FeO & Mistura (g) \\
\hline M1 & 71,6 & 25,6 & 1,65 & 0,68 & 0,13 & 0,34 & 0,36 & 65,0 \\
M2 & 65,8 & 31,5 & 1,60 & 0,64 & 0,15 & 0,31 & 0,48 & 65,0 \\
M3 & 60,0 & 37,4 & 1,51 & 0,63 & 0,18 & 0,28 & 0,62 & 65,0 \\
M4 & 56,1 & 41,3 & 1,49 & 0,61 & 0,25 & 0,27 & 0,73 & 65,0 \\
\hline
\end{tabular}

\subsection{Equilíbrio do gusa com a mistura inicial}

Para a simulação do equilíbrio também foi utilizado o software FactSage 6.4, como banco de dados foi utilizado FtOxid e FactPS e sub banco de dados Slag?, Fe-liquid e Pure solids para as temperaturas $\mathrm{T} 1=1350^{\circ} \mathrm{C}, \mathrm{T} 2=1400^{\circ} \mathrm{C}$ e $\mathrm{T} 3=1450^{\circ} \mathrm{C}$.

\section{RESULTADOS DOS EXPERIMENTOS}

Os gráficos abaixo apresentam o resultado dos testes experimentais feito por Broseghini [2] para as misturas apresentadas. Estão nele relacionados à razão entre a porcentagem de fosforo final e inicial versus o tempo, e no segundo gráfico mostra a eficiência de cada uma dessas misturas. A eficiência é calculada com base na seguinte equação

$$
\eta(\%) D e-P=\frac{[P]_{\text {inicial }}-[P]_{\text {final }}}{[P]_{\text {inicial }}} x 100
$$


Segue então os resultados:
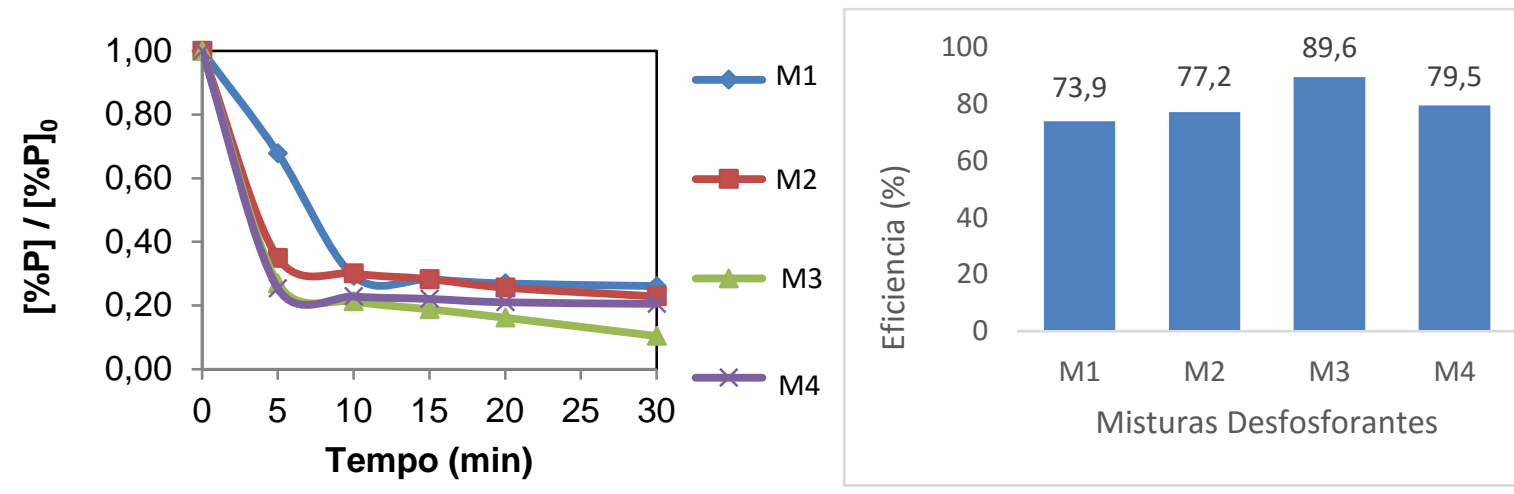

Figura 1. (a) Porcentagem de fosforo inicial x tempo e (b) Eficiência x Mistura

Como podemos observar pelo primeiro gráfico o tempo da reação de desfosforação ocorre nos primeiros 10 minutos. No segundo gráfico observamos que a mistura com melhor desempenho foi a M3 com $89,6 \%$ de eficiência. Assim, espera-se que para menores temperaturas a eficiência das misturas aumente. Para isso, fez-se a analise através dos softwares de termodinâmica.

\subsection{Avaliação da eficiência da desfosforação através das misturas iniciais}

Primeiramente, avaliou-se o processo de desfosforação com os resultados obtidos no estudo da mistura inicial. A avaliação foi feita por modelos da literatura e também através de softwares de termodinâmica.

\subsubsection{Avaliação com modelos literários}

Os cálculos necessários para a avaliação á partir de modelos literários, foram feitos com base nas equações mostradas a seguir anteriormente apresentadas por Healy [3] e Suíto[4].

Tabela 2. Modelos de coeficientes de partição [2] Autor Modelo

\begin{tabular}{rr}
\hline Young & $\log C p=-18,184+35,84 . \Lambda-22,35 \cdot \Lambda^{2}+\frac{22.930 . \Lambda}{T}-0,06257 . \% F e O$ \\
& $-0,04256 . \% M n O+0,359 . \% P_{2} O_{5}^{0,3}$ \\
\hline Healy & $\log \frac{(\% P)}{[\% P]}=\frac{22.350}{T}+0,08 \cdot(\% C a O)+2,5 \cdot \log \left(\% F e_{\text {total }}\right)-16$ \\
\hline $\begin{array}{c}\text { Suito e } \\
\text { Inoue }\end{array}$ & $\log \frac{(\% P)}{[\% P]}=0,072[(\% \mathrm{CaO})+0,3 .(\% \mathrm{MgO})]+2,5 \cdot \log \left(\% F e_{\text {total }}\right)+\frac{11.570}{T}-10,52$ \\
\hline
\end{tabular}

Ainda tem-se o coeficiente de atividade do $\mathrm{P}_{2} \mathrm{O}_{5}\left({ } \mathrm{P}_{2} \mathrm{O}_{5}\right)$, (Equação 8):

$$
\log \left(\gamma P_{2} O_{5}\right)=-1,22 .\left(22 . . N_{\mathrm{CaO}}+15 . . N_{\mathrm{MgO}}+13 . N_{\mathrm{MnO}}+12 . N_{\mathrm{FeO}}-2 . N_{\mathrm{SiO} 2}\right)-\frac{42000}{T}+23,58
$$

A basicidade de cada óxido é calculada em função da eletronegatividade de Pauling e a basicidade teórica da escória é dada pela média ponderada das contribuições individuais, através da fração molar. Assim temos pela (Equação 9):

$$
\wedge_{i}=\frac{0,74}{(X-0,26)}
$$


Onde $\mathrm{X}$ é a eletronegatividade de Pauling, $\mathrm{N}$ fração molar do óxido ou fluoreto na escória, $\wedge$ ibasicidade de cada óxido e $\Lambda_{\text {esc }}$ basicidade da escória que é calculada:

$$
\wedge_{e s c}=\sum \wedge_{i} N
$$

A tabela abaixo (Tabela 3) mostra os resultados obtidos a partir das equações acima, para cada mistura em cada uma das temperaturas.

Tabela 3. Basicidade ótica, coeficiente de atividade do $\mathrm{P}_{2} \mathrm{O}_{5}$, capacidade de fosfato e coeficiente de partição do fósforo das misturas desfosforantes

\begin{tabular}{|c|c|c|c|c|c|c|c|}
\hline Temp. & Mistura & $\Lambda$ & $\begin{array}{l}\text { log CP } \\
\text { Young }\end{array}$ & $\begin{array}{c}\text { LP } \\
\text { Healy }\end{array}$ & $\begin{array}{c}\text { LP } \\
\text { Suito }\end{array}$ & у P2O5 & $\eta$ \\
\hline $1350^{\circ} \mathrm{C}$ & & 0,66 & 0,58 & 4,18 & 2,82 & $2,49 E-15$ & \\
\hline $1400^{\circ} \mathrm{C}$ & M1 & 0,66 & 0,3 & 3,77 & 2,61 & $1,48 \mathrm{E}-14$ & 73,9 \\
\hline $1450^{\circ} \mathrm{C}$ & & 0,66 & 0,03 & 3,38 & 2,4 & $7,90 \mathrm{E}-14$ & \\
\hline $1350^{\circ} \mathrm{C}$ & & 0,69 & 1,56 & 4,56 & 3,15 & $4,08 \mathrm{E}-16$ & \\
\hline $1400^{\circ} \mathrm{C}$ & $\mathrm{M} 2$ & 0,69 & 1,27 & 4,15 & 2,94 & $2,42 \mathrm{E}-15$ & 77,2 \\
\hline $1450^{\circ} \mathrm{C}$ & & 0,69 & 1 & 3,76 & 2,74 & $1,30 \mathrm{E}-14$ & \\
\hline $1350^{\circ} \mathrm{C}$ & & 0,72 & 2,49 & 4,93 & 3,48 & $5,42 \mathrm{E}-17$ & \\
\hline $1400^{\circ} \mathrm{C}$ & M3 & 0,72 & 2,19 & 4,52 & 3,26 & $3,22 \mathrm{E}-16$ & 89,6 \\
\hline $1450^{\circ} \mathrm{C}$ & & 0,72 & 1,9 & 4,14 & 3,06 & $1,72 \mathrm{E}-15$ & \\
\hline $1350^{\circ} \mathrm{C}$ & & 0,74 & 3,08 & 5,17 & 3,69 & $1,75 \mathrm{E}-17$ & \\
\hline $1400^{\circ} \mathrm{C}$ & M4 & 0,74 & 2,77 & 4,76 & 3,47 & $1,04 \mathrm{E}-16$ & 79,5 \\
\hline $1450^{\circ} \mathrm{C}$ & & 0,74 & 2,47 & 4,37 & 3,27 & $5,55 \mathrm{E}-16$ & \\
\hline
\end{tabular}

De acordo com outro estudo e experimento feito por Broseghini [2] sobre as misturas a uma temperatura de $1400^{\circ} \mathrm{C}$, temos os seguintes resultados que relacionam a eficiência desfosforante com os parâmetros termodinâmicos obtidos. Os resultados para basicidade e coeficiente de atividade do $\mathrm{P}_{2} \mathrm{O}_{5}$ relacionados com a eficiência estão apresentados na Figura 2:

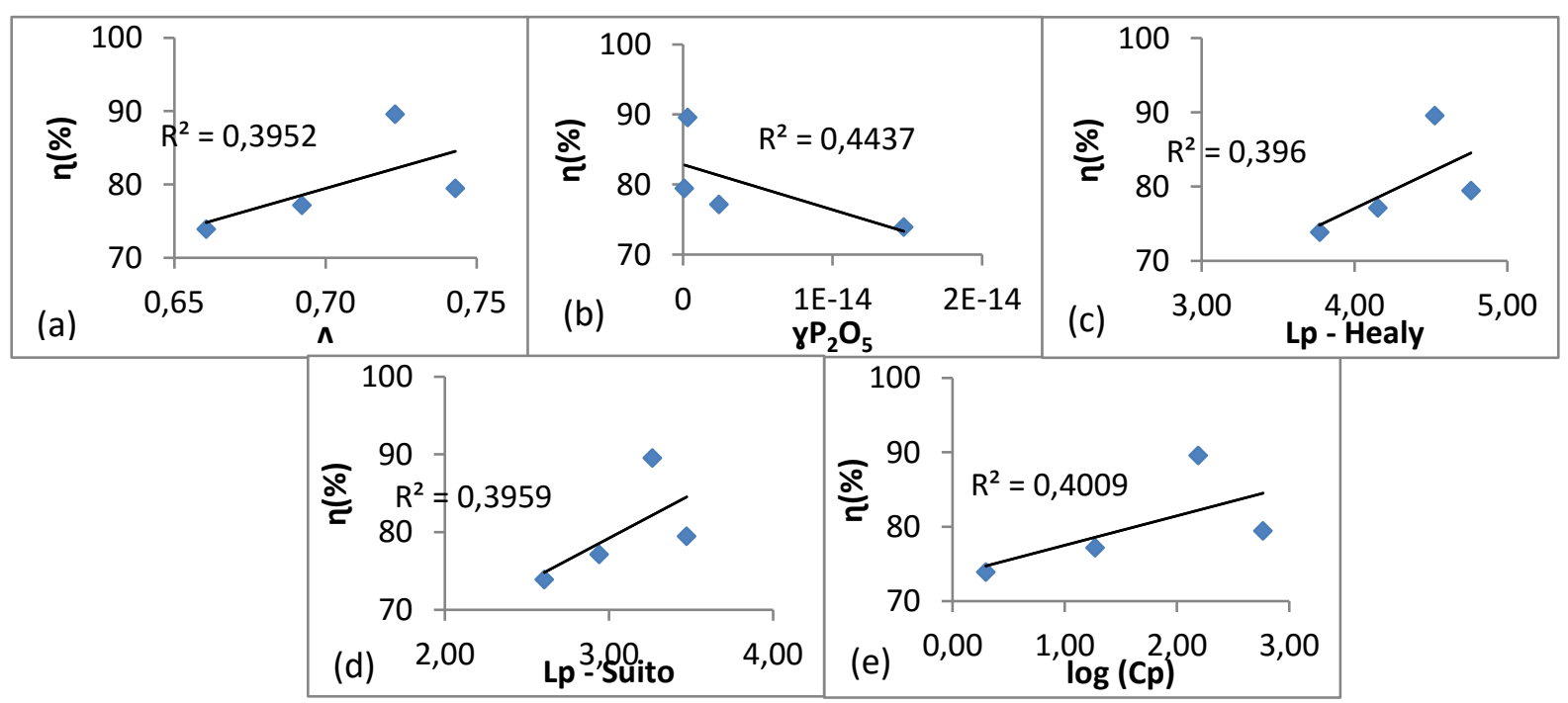

Figura 2. Relação entre eficiência e (a)basicidade; (b)coeficiente de atividade do $\mathrm{P}_{2} \mathrm{O}_{5}$; (c) Lp modelo Healy; (d)Lp modelo Suito e (e) log do coeficiente de partição do fósforo. 
Com esse estudo feito anteriormente, pode-se perceber que com o aumento da basicidade ótica, a diminuição do coeficiente de atividade $\mathrm{P}_{2} \mathrm{O}_{5}, 0$ aumento da capacidade de fosfato ou ainda do coeficiente de partição do fósforo da mistura, aumenta-se também a eficiência desfosforante.[2], mostrando assim uma tendência de um cenário favorável ao processo. Observa-se que a correlação entre os parâmetros termodinâmicos e a eficiência obtida nos testes experimentais foi relativamente baixo, aproximadamente 0,4 . Tal fato é justificado pela limitação de fatores cinéticos e características do processo de desfosforação que são levados em consideração no cálculo destes parâmetros.

Pode-se ainda relacionar os coeficientes obtidos com a temperatura, para avaliar a influencia da mesma.

A partir dos dados mostrados na tabela acima, (Tabela 3) pode-se perceber que os melhores valores para os parâmetros termodinâmicos (maiores valores de basicidade, menores valores de coeficiente de atividade do $\mathrm{P}_{2} \mathrm{O}_{5}$, maiores valores de Cp e Lp ) são obtidos a partir das menores temperaturas.

\subsubsection{Avaliação com uso de softwares de termodinâmica}

A fim de obter maiores informações, a avaliação das misturas iniciais também foi feita através do software FactSage para comparação com os dados termodinâmicos anteriormente calculados.

As misturas foram inseridas no software para as três temperaturas $\mathrm{T} 1=1350^{\circ} \mathrm{C}$, $\mathrm{T} 2=1400^{\circ} \mathrm{C}$ e $\mathrm{T} 3=1450^{\circ} \mathrm{C}$ para verificar a quantidade de fase liquida, a possibilidade de formação de compósitos sólidos e obtenção das atividades de $\mathrm{CaO}$ e $\mathrm{FeO}$.

Todos os dados acima citados estão expressos na tabela a seguir. Vale ressaltar que os valores mostrados de eficiência desfosforante foram obtidos de estudos anteriores feitos com as mesmas misturas a temperatura de $1400^{\circ} \mathrm{C}$.

Tabela 4. Resultados obtidos a partir do aquecimento das misturas iniciais a diferentes temperaturas do processo, no software FactSage.

\begin{tabular}{|c|c|c|c|c|c|c|c|c|}
\hline Temp & Mistura & \%Líquido & $\% \mathrm{CaO}$ & $\mathrm{aCaO}$ & aFeO & Visc* & Visc** & $\eta$ \\
\hline $1350^{\circ} \mathrm{C}$ & \multirow{3}{*}{ M1 } & 100 & 0 & 0,44 & 0,63 & 0,258 & 0,258 & - \\
\hline $1400^{\circ} \mathrm{C}$ & & 100 & 0 & 0,4 & 0,63 & 0,227 & 0,227 & 73,88 \\
\hline $1450^{\circ} \mathrm{C}$ & & 100 & 0 & 0,36 & 0,63 & 0,201 & 0,201 & - \\
\hline $1350^{\circ} \mathrm{C}$ & \multirow{3}{*}{ M2 } & 100 & 0 & 0,7 & 0,51 & 0,301 & 0,301 & - \\
\hline $1400^{\circ} \mathrm{C}$ & & 100 & 0 & 0,63 & 0,51 & 0,26 & 0,260 & 77,15 \\
\hline $1450^{\circ} \mathrm{C}$ & & 100 & 0 & 0,57 & 0,51 & 0,227 & 0,227 & - \\
\hline $1350^{\circ} \mathrm{C}$ & \multirow{3}{*}{ M3 } & 97,44 & 2,56 & 1 & 0,41 & 0,349 & 0,381 & - \\
\hline $1400^{\circ} \mathrm{C}$ & & 99,33 & 0,67 & 1 & 0,38 & 0,297 & 0,304 & 89,57 \\
\hline $1450^{\circ} \mathrm{C}$ & & 100 & 0 & 0,93 & 0,38 & 0,255 & 0,255 & - \\
\hline $1350^{\circ} \mathrm{C}$ & \multirow{3}{*}{ M4 } & 91,46 & 8,56 & 1 & 0,41 & 0,385 & 0,523 & - \\
\hline $1400^{\circ} \mathrm{C}$ & & 93,23 & 6,79 & 1 & 0,38 & 0,324 & 0,412 & 79,46 \\
\hline $1450^{\circ} \mathrm{C}$ & & 95,01 & 5,01 & 1 & 0,36 & 0,275 & 0,328 & - \\
\hline
\end{tabular}

Para a temperatura de $1400^{\circ} \mathrm{C}$ temos os seguintes gráficos relacionados com a eficiência mostrados na (Figura 3): 

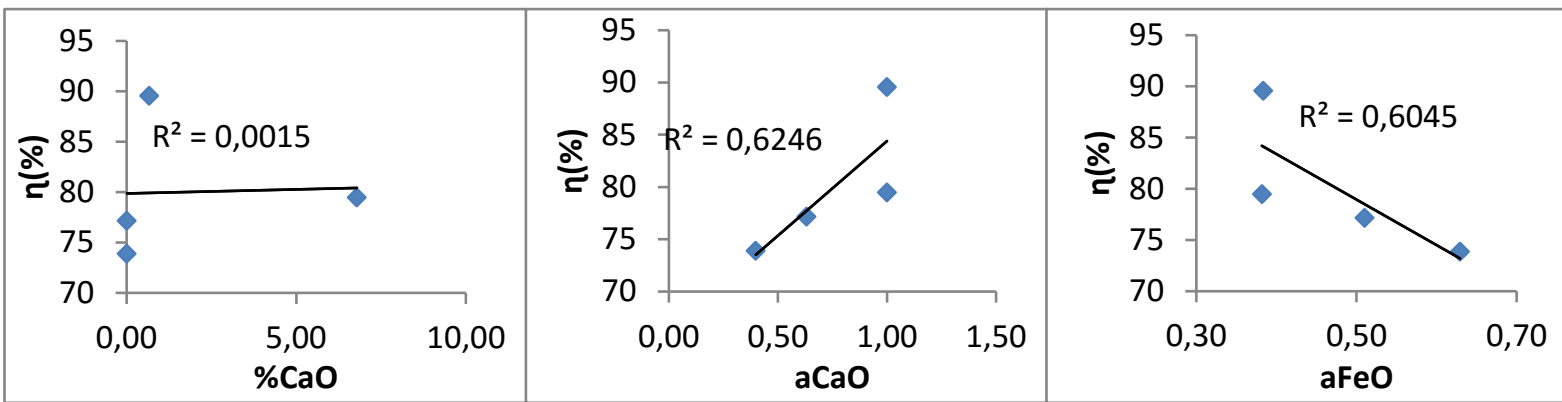

Figura 3. Relação entre eficiência e (a)\%CaO sólido, (b)atividade $\mathrm{CaO}$ e (c) atividade $\mathrm{FeO}$

Observa-se através dos gráficos que a diminuição da quantidade de líquido aliada a um aumento de atividade do $\mathrm{CaO}$ gera um consequente aumento na eficiência da mistura desfosforante.[2] Por outro lado, através do gráfico \%Líquido $x \% \mathrm{CaO}$ percebe-se que há um ponto onde esses valores passam a prejudicar a eficiência. $O$ aumento de $\mathrm{CaO}$ diminui a quantidade de líquido da escória e como a escoria líquida favorece a reação, quando esse liquido diminui, acaba prejudicando o processo.

Já em relação à temperatura, podemos dizer que a relação entre a \% $\mathrm{CaO}$ e a temperatura é a seguinte: maiores temperaturas há menores porcentagens da substância, já a porcentagem de líquido aumenta com o aumento da temperatura, o que poderia sugerir uma maior eficiência em maiores temperaturas.

\subsection{Avaliação da eficiência da desfosforação através das escórias finais}

\subsubsection{Avaliação com modelos de coeficiente de atividade $\mathrm{P}_{2} \mathrm{O}_{5}$, capacidade de fosfato e coeficiente de partição do fósforo}

Da mesma forma que calculamos os coeficientes termodinâmicos para as misturas iniciais, foram calculados para as escórias finais para a avaliação da eficiência com base em modelos literários. Para uma avaliação mais clara e eficiente do processo relacionado a diferentes temperaturas foi feita uma avaliação da porcentagem de fósforo no equilíbrio. Os dados obtidos estão apresentados na seguinte tabela.

Tabela 5. Coeficientes termodinâmicos escória final

\begin{tabular}{|c|c|c|c|c|c|c|c|c|}
\hline Temp. & Mistura & $\Lambda$ & $\begin{array}{l}\text { log CP } \\
\text { Young }\end{array}$ & $\begin{array}{c}\text { LP } \\
\text { Healy }\end{array}$ & $\begin{array}{c}\text { LP } \\
\text { Suito }\end{array}$ & у Р205 & $\%$ Peq & $\eta$ \\
\hline $1350^{\circ} \mathrm{C}$ & & 0,71 & 3,9 & 4,15 & 2,81 & $1,69 E+16$ & $3,03 E-05$ & \\
\hline $1400^{\circ} \mathrm{C}$ & M1 & 0,71 & 3,6 & 3,74 & 2,6 & $1,00 \mathrm{E}+17$ & 4,79E-05 & 73,9 \\
\hline $1450^{\circ} \mathrm{C}$ & & 0,71 & 3,32 & 3,35 & 2,4 & $5,38 E+17$ & $9,45 \mathrm{E}-05$ & \\
\hline $1350^{\circ} \mathrm{C}$ & & 0,75 & 5,06 & 4,65 & 3,21 & $2,1 \mathrm{E}+13$ & $1,34 \mathrm{E}-06$ & \\
\hline $1400^{\circ} \mathrm{C}$ & M2 & 0,75 & 4,74 & 4,24 & 2,99 & $1,2 \mathrm{E}+14$ & $3,12 E-06$ & 77,2 \\
\hline $1450^{\circ} \mathrm{C}$ & & 0,75 & 4,45 & 3,85 & 2,79 & $6,6 E+14$ & $7,50 \mathrm{E}-06$ & \\
\hline $1350^{\circ} \mathrm{C}$ & & 0,81 & 6,37 & 5,29 & 3,75 & $3,13 E+06$ & 1,39E-06 & \\
\hline $1400^{\circ} \mathrm{C}$ & M3 & 0,81 & 6,03 & 4,88 & 3,53 & $1,86 \mathrm{E}+07$ & 3,31E-06 & 89,6 \\
\hline $1450^{\circ} \mathrm{C}$ & & 0,81 & 5,71 & 4,49 & 3,33 & $9,96 \mathrm{E}+07$ & $9,12 \mathrm{E}-06$ & \\
\hline $1350^{\circ} \mathrm{C}$ & & 0,82 & 6,81 & 5,53 & 3,94 & $2,76 \mathrm{E}+07$ & $1,4 \mathrm{E}-06$ & \\
\hline $1400^{\circ} \mathrm{C}$ & M4 & 0,82 & 6,46 & 5,12 & 3,73 & $1,64 \mathrm{E}+08$ & $3,4 \mathrm{E}-06$ & 79,5 \\
\hline $1450^{\circ} \mathrm{C}$ & & 0,82 & 6,13 & 4,73 & 3,53 & $8,77 E+08$ & $8,8 \mathrm{E}-06$ & \\
\hline
\end{tabular}


Como pode se observar na Tabela 5, os menores teores de fósforo no equilíbrio foram obtidos em menores temperaturas de trabalho, além disso, observa-se que se obteve os maiores valores de coeficientes de partição e capacidade de fosfato para menores temperaturas. Entretanto, analisando a eficiência de desfosforação, aquele que apresentou maior coeficiente de partição (M4), não foi aquele que apresentou maior eficiência.

Nas figuras abaixo, figura 5 e figura 6 , estão sendo relacionados os valores obtidos com a eficiência obtida em testes experimentais de trabalhos passados, Broseghini [2].

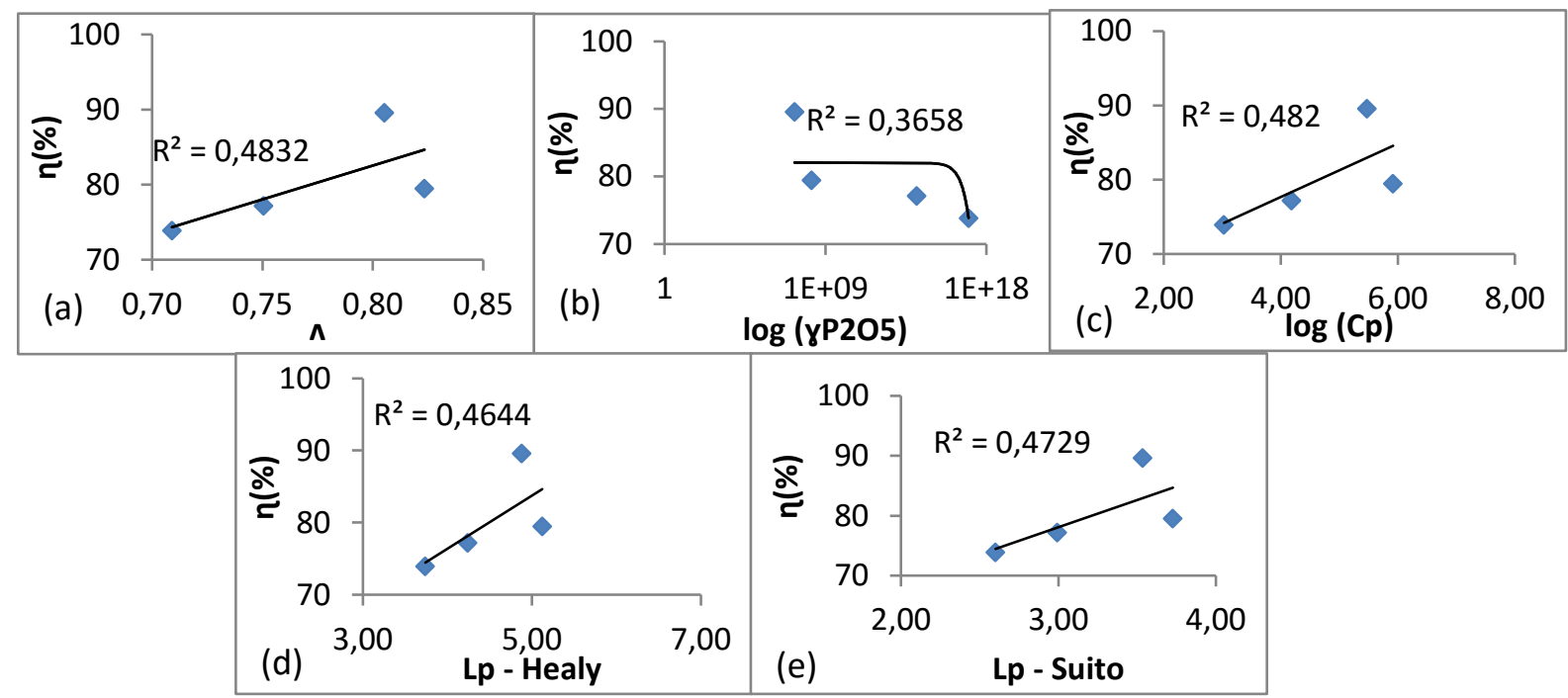

Figura 4. Relação entre eficiência e (a) basicidade; (b) $\log \mathrm{\gamma}_{2} \mathrm{O}_{5}$; (c)log $\mathrm{Cp}$; (d) Lp Healy (e) Lp Suito

Como se observa pelos gráficos, não é possível fazer uma boa correlação com os dados termodinâmicos apresentados, com a eficiência desfosforante. Portanto esses parâmetros não são precisos para prever eficiência. Isso pode ser justificado porque os coeficientes se referem a termodinâmica da reação e não avaliam a cinética da mesma.

\subsubsection{Avaliação com uso de software}

Nessa etapa, a escória final obtida no equilíbrio, feito nas simulações é estudada para avaliar a desfosforação através dos cálculos feitos pelo software das fases presentes na escória. Para isso foram feitas duas considerações importantes: Fases que apareceram no equilíbrio, mas não são formadas em casos reais foram inibidas e a formação do gás CO também foi inibida.

O FactSage além das fases e atividades também calcula a viscosidade da mistura; porém, somente da fase líquida, por isso é necessário fazer uma correção através da expressão (Equação 11) de Einstein-Roscoe [5] mostrada abaixo:

$$
\eta_{e}=\eta \cdot\left(1-1,35 \cdot x_{s o l}\right)^{-\frac{5}{2}}
$$

Os dados obtidos são mostrados na tabela (Tabela 6) abaixo: 
Tabela 6. Fases formadas e atividade de $\mathrm{CaO}$ e FeO na escória final nas temperaturas de teste obtidas a partir do FactSage.

\begin{tabular}{|c|c|c|c|c|c|c|c|c|c|c|}
\hline \multirow[b]{2}{*}{ Temp } & \multirow[b]{2}{*}{ Mist. } & \multirow[b]{2}{*}{ \%Líq. } & \multicolumn{3}{|c|}{ Fases sólidas formadas (\%) } & \multirow{2}{*}{$\mathrm{aCaO}$} & \multirow[b]{2}{*}{ aFeO } & \multirow[b]{2}{*}{ Visc.* } & \multirow[b]{2}{*}{ Visc.** } & \multirow[b]{2}{*}{$\eta(\%)$} \\
\hline & & & $\mathrm{CaO}$ & 3CaO.P2O5 & $\mathrm{MgO}$ & & & & & \\
\hline T1 & \multirow{3}{*}{ M1 } & 96,39 & 0 & 3,61 & 0 & 0,1 & 0,78 & 0,55 & 0,62 & $\sim$ \\
\hline T2 & & 97,86 & 0 & 2,14 & 0 & 0,1 & 0,77 & 0,45 & 0,48 & 73,88 \\
\hline T3 & & 99,52 & 0 & 0,48 & 0 & 0,09 & 0,77 & 0,37 & 0,38 & $\sim$ \\
\hline T1 & \multirow{3}{*}{ M2 } & 100 & 0 & 0 & 0 & 0,27 & 0,61 & 0,67 & 0,67 & $\sim$ \\
\hline $\mathrm{T} 2$ & & 100 & 0 & 0 & 0 & 0,26 & 0,6 & 0,54 & 0,54 & 77,15 \\
\hline T3 & & 100 & 0 & 0 & 0 & 0,24 & 0,59 & 0,44 & 0,44 & $\sim$ \\
\hline T1 & \multirow{3}{*}{ M3 } & 92,7 & 6,67 & 0 & 0,63 & 1 & 0,31 & 0,78 & 1,01 & $\sim$ \\
\hline $\mathrm{T} 2$ & & 93,8 & 5,81 & 0 & 0,4 & 1 & 0,29 & 0,61 & 0,76 & 89,57 \\
\hline T3 & & 94,99 & 4,88 & 0 & 0,13 & 1 & 0,26 & 0,49 & 0,59 & $\sim$ \\
\hline T1 & \multirow{3}{*}{ M4 } & 85,84 & 14,09 & 0 & 0,07 & 1 & 0,32 & 0,82 & 1,4 & $\sim$ \\
\hline T2 & & 86,74 & 13,26 & 0 & 0 & 1 & 0,3 & 0,65 & 1,06 & 79,46 \\
\hline T3 & & 87,67 & 12,33 & 0 & 0 & 1 & 0,27 & 0,51 & 0,81 & $\sim$ \\
\hline
\end{tabular}

*Viscosidade calculada através do FactSage. ${ }^{* *}$ Viscosidade corrigida

Com a tabela, observa-se que É necessário um cenário favorável de todas essas variáveis para 0 melhor fator ser encontrado. Por isso para algumas misturas, mesmo com temperaturas mais baixas, observa-se que o fator de desfosforação diminuiu devido ao fato de que as outras variáveis não se encontram em seus valores ideias, como por exemplo, a mistura 1 (M1) em que a atividade da $\mathrm{CaO}$ está bem abaixo do ideal $(\mathrm{aCaO}=1)$.Já as misturas $\mathrm{M} 3$ e $\mathrm{M} 4$ apresentam valores consideráveis de fase sólida formada, e de acordo com a equação cinética (Equação 6), quando a escória apresenta fase solida a reação de desfosforação e prejudicada uma vez que diminui a interface metal/escoria.

Podemos ainda relacionar a temperatura com a viscosidade e a \%Líquido. Essa relação está apresentada na figura 5 abaixo:

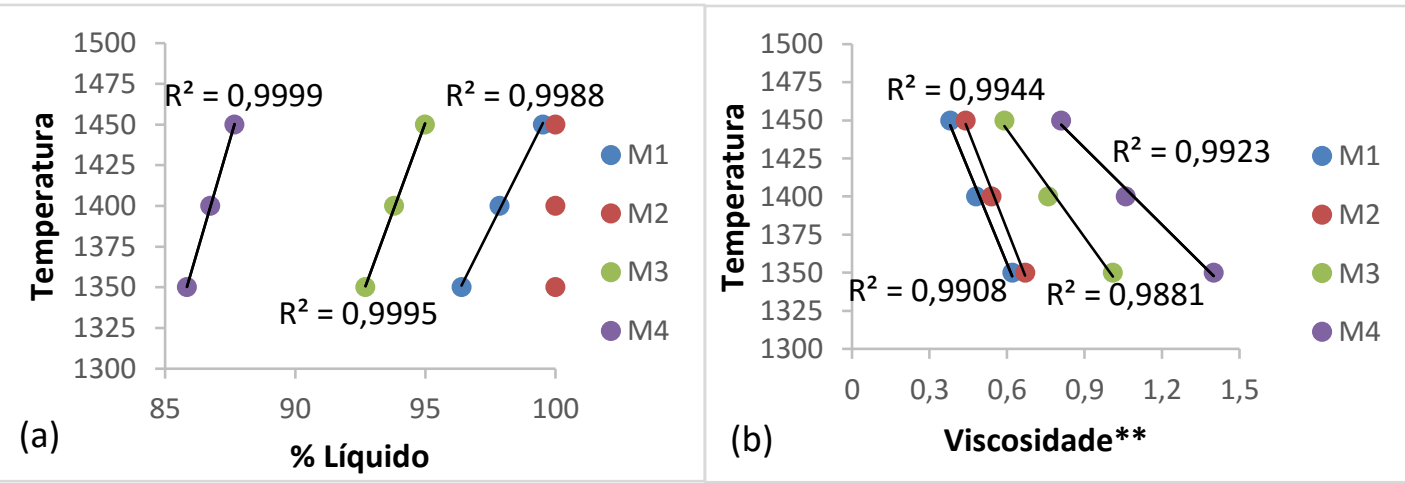

Figura 5. Relação entre temperatura e (a) \%Líquido e (b) Viscosidade**

Através dos gráficos vemos uma boa correlação entre temperatura e \%Líquido e temperatura e viscosidade. A relação entre temperatura e viscosidade e temperatura é diretamente proporcional, enquanto que a relação temperatura $x$ viscosidade é inversa, com o aumento da temperatura observamos a diminuição da viscosidade. 


\section{CONCLUSÃO}

Sendo assim, com base na literatura afirma-se que os maiores valores de eficiência estão associados aos maiores valores de $\mathrm{Cp}$, $\mathrm{Lp}$ e basicidade associada aos menores valores de atividade do $\mathrm{P}_{2} \mathrm{O}_{5}$. De forma análoga, o melhores valores para esses fatores estão relacionados com as menores temperaturas. Porém, com as análises feitas a partir do software termodinâmico conclui-se que é necessário buscar a temperatura onde os melhores valores para as atividades do $\mathrm{CaO}$ e $\mathrm{FeO}$ além da maior \%Líquido na escória sejam obtidos a fim de conquistar os melhores valores para FDeP e consequentemente os melhores valores para eficiência do processo

\section{Agradecimentos}

A CAPES pelo apoio ao projeto através do edital PRPPG 05/2015.

\section{REFERÊNCIAS}

1 TURKDOGAN, E. T. Slag Composition Variations Causing Variations in Steel Dephosphorisation and Desulphurisation in Oxygen Steelmaking. ISIJ International, $\mathrm{n}$. 9, v. 40 , p. $827-832,2000$.

2 BROSEGHINI, F. C. Utilização de termodinâmica computacional no estudo da desfosforação de ferro-gusa por escórias do sistema CaO-FeO. 2015.106 f. Dissertação (Pós Graduação em Engenharia Metalúrgica e de Materiais), Instituto Federal do Espírito Santo, Espírito Santo, 2015.

3 HEALY, G. A new look at phosphorus distribution. Journal of the Iron and Steel Institute, v. 208, p. 664-668, 1970.

4 SUITO, M. et al. Total hot metal pretreatment system at Kawasaki steel. Kawasaki Steel Technical Review, v. 88, p. 18-27, 2003.

5 EINSTEIN, A. Eine neue bestimmung der molekudimensionnen. Annales de Physique, v. 19, p. 289-306), 1906. 